Cite as: Stephane H Maes, (2020), "Derivation of the Equivalence Principle in a Multi-fold Universe", viXra:2010.0090v1, https://vixra.org/pdf/2010.0090v1.pdf, https://shmaesphysics.wordpress.com/2020/06/29/ derivation-of-the-equivalence-principle-in-a-multi-fold-universe/, June 19, 2020.

\title{
Derivation of the Equivalence Principle in a Multi-fold Universe
}

\author{
Stephane H. Maes ${ }^{1}$
}

June 29, 2020

\begin{abstract}
:
In a multi-fold universe, gravity emerges from Entanglement through the multi-fold mechanisms. As a result, gravity-like effects appear in between entangled particles that they be real or virtual. Long range, massless gravity results from entanglement of massless virtual particles. Entanglement of massive virtual particles leads to massive gravity contributions at very small scales. Multi-folds mechanisms also result into a spacetime that is discrete, with a random walk fractal structure and non-commutative geometry that is Lorentz invariant and where spacetime nodes and particles can be modeled with microscopic black holes. All these recover General relativity at large scales and semi-classical model remain valid till smaller scale than usually expected. Gravity can therefore be added to the Standard Model. This can contribute to resolving several open issues with the Standard Model.

The mechanisms proposed to address entanglement and that are responsible for gravity when considering entanglement of virtual particles, also automatically result into the (weak) principle of equivalence, without postulating it. It also required better qualifying the tenancy model of multi-folds in terms of Higgs interactions.
\end{abstract}

\section{Introduction}

The new preprint [1] proposes contributions to several open problems in physics like the reconciliation of General Relativity (GR) with Quantum Physics, explaining the origin of gravity proposed as emerging from quantum (EPREinstein Podolsky Rosen) entanglement between particles, detailing contributions to dark matter and dark energy and explaining other Standard Model mysteries without requiring New Physics beyond the Standard Model other than the addition of gravity to the Standard Model Lagrangian. All this is achieved in a multi-fold universe that may well model our real universe, which remains to be validated.

With the proposed model of [1], spacetime and Physics are modeled from Planck scales to quantum and macroscopic scales and semi classical approaches appear valid till very small scales. In [1], it is argued that spacetime is discrete, with a random walk-based fractal structure, fractional and noncommutative at, and above Planck scales (with a 2-D behavior and Lorentz invariance preserved by random walks till the early moments of the universe). Spacetime results from past random walks of particles. Spacetime locations and particles can be modeled as microscopic blackholes (Schwarzschild for photons and spacetime coordinates, and metrics between Reisner Nordstrom [2] and Kerr Newman [3] for massive and possibly charged particles - the latter being possibly extremal). Although surprising, [1] recovers results consistent with other like [4], while also being able to justify the initial assumptions of black holes from the gravity or entanglement model in a multi-fold universe. The resulting gravity model recovers General Relativity at larger scale, as a 4-D process, with massless gravity, but also with massive gravity components at very small scale that make gravity significant at these scales. Semi-classical models also turn out to work well till way smaller scales that usually expected.

\footnotetext{
${ }^{1}$ shmaes.physics@gmail.com
} 
In this paper, we remain at a high level of discussion of the analysis and references are generic for the subjects. It makes the points accessible to a wider audience and keeps the door open to further papers or discussions devoted to details of interest. Yet, it requires the reader to review [1], as we do not revisit here all the details of the multifold mechanisms or reconstruction of spacetime. More targeted references for all the material discussed here are compiled in [1].

\section{The Equivalence Principles}

The equivalence principle reflects how Einstein managed to first conceive, then formalize the extension of Special Relativity to any observer, no matter what his location and velocity and in particular to understand that nothing distinguish (non-gravitational) Physics in the presence of gravity from Physics in its absence but in an accelerated reference frame [5].

Rigorously, the principle exists in at least 3 forms:

- The weak equivalence principle that states that inertial mass and gravity mass (i.e. the charge for the gravitation interaction) are the same.

- Einstein's equivalence principle, which really states the intuitive consideration above as the principle that any non-gravitational physics in a free-falling laboratory is independent of the velocity of the laboratory and its location in spacetime

- The strong equivalence principle which adds to Einstein's equivalence principle that gravitational physics then depends only on the initial conditions, not the constitution of the (small) entities.

At the core, there are 3 key underlying principles: i) inertial mass = gravitational mass ii) (General) covariance (also related to background independence) iii) gravity and acceleration are equivalent in the sense that they can play the role on one another in different suitable frames.

These principles + Special relativity and Einstein's genius gave us GR!

Then, two other giants of Physics provided (Quantum Field Theory) QFT derivations of the weak equivalence principle:

- $\quad$ Steven Weinberg showed that the Lorentz invariance of the scattering S-matrix involving gravitons ensures that the inertial mass and the gravitational mass must be the same [6]. As a side note, Weinberg then went on showing that Lorentz invariant S-Matrix for covariance and spin-2 long range (massless) interactions automatically recovers GR [7].

- $\quad$ Richard Feynman independently reconstructed GR from first principles and QFT (propagator and Feynman diagrams) showing that gravity must be Lorentz invariant, mediated by massless with spin-2 gravitons and recovers the equivalence principle as well as covariance / Gauge invariance of Fierz-Pauli [9].

As [1], recovers GR / massless gravity as well as additional massive gravity and entanglement contributions, from completely different considerations related to the EPR paradox, it is of interest to see:

- Can we recover the equivalence principle, for GR?

- Does it extend beyond GR?

- $\quad$ Are there any more insights from this analysis?

\section{The Equivalence principles in a Multi-fold universe for Massless Gravity}

Intuitively, the recovery of GR should imply that we recover the equivalence principles; or [1] assertions would be suspicious. 
If we go back to the basics of [1], the multi-fold mechanisms have folds activated when EPR entanglement takes place. As a result, bundles of folds are activated and paths of particles crossing the support domains of the folds have paths mapped to each fold, resulting (per fold) into an attractive effective potential in $\frac{1}{r^{2}}$ between the entangled particles and towards their center of mass (integration over all relevant folds in a multi-fold leads to potentials in $\frac{1}{r}$. Each fold contributes its Ricci curvature scalar (GR is recovered by adding all these Ricci scalars and Ricci Tensors (by adding direction of attraction for every point of spacetime). The effective potential is the result of computing the path integral contribution of the path on the activated folds. It is also proportional to the mass of the particle on the folds.

So the resulting Lagrangian in [1] contains the Lagrangian of the particle, without gravity and with the inertial mass, plus a potential that reconstructs GR or Newton gravity with the same inertial mass: gravitational mass and inertial masses is a direct result of the multi-fold mechanisms. We have recovered the weak equivalence mechanism in multi-fold universes. Symmetries between flipping the roles of the particles also lead to determining that the effective potential will also be proportional to the mass of the source ([1] presents a different also valid argument for that).

Because GR is recovered at large scales and multi-fold mechanisms are covariant (e.g. based on path integrals) respecting Lorentz symmetries and background independence, we also recover Einstein's equivalence principle and the strong equivalence principle.

\section{Beyond Massless Gravity}

Because multi-fold universe have also massive gravity contributions, at very small scales, we also need to note that the reasoning of section 3 can be repeated as is, for these contributions within their ranges. So the respect of the equivalence principle extends to all gravity contributions in multi-fold universes.

Entanglement (e.g. between real particles) is conventionally not counted as gravity effects. Yet they bring gravity like contributions in multi-fold universes $[1,10]$. Again the same mechanisms apply with the same reasoning: the weak equivalence principle extends to entanglement gravity-like effects.

On the other hand, the ranges and anisotropy / symmetry breaking introduced by two EPR entangled particles does not allow the same generic considerations for the two other variations of the principle: it may depend on the use case (we already know they considerations works for all the gravity use cases where the effects come from virtual EPR pairs). It may not really matter as we probably would consider that entanglement (between real particles) is not gravity.

\section{A Quick Note on Fold Tenancy and Higgs Mechanisms Impact}

[1] proposes multi-fold mechanisms where folds and mapping are multi-tenant with strong partitioning, so that only one particles is present per fold instance and no interaction takes place other than at entry and exit.

The analysis above necessitate to detail a bit more this statement. It is correct if the Lagrangian on the fold is the Lagrangian of the particle in spacetime, without other interactions. This implies that, the folds are spacetime also, just curved. Therefore the tenancy model is to be understood as applying for particles. A uniform field in spacetime and tied to it would also be present and interact in a fold. So Higgs and the Higgs field are expected to be present in the multi-folds and as a result the particle propagator remains associated to their mass in the folds. Note that if inflation was to be described with an inflaton field, it probably is not be present in the multi-folds as their 
dynamics is already said by the moves of the entangled particles (real and virtual) or field. These already take inflaton into account [11].

This model can be motivated by the fact that otherwise, particles could mutate in ways not intended by the mechanisms designed to solve EPR paradoxes and no observed in practice. Otherwise, for example, electroweak symmetry breaking and Englert-Brout-Higgs-Guralnik-Hagen-Kibble mechanisms would not be in the multi-folds and physics would be different. [1] was clear that physics is the same except without particle to particle interactions; which we must explicitly now qualify as not excluding Higgs interactions with the particles in the multi-fold.

This multi-fold tenancy model can also be motivated by how multi-fold and mappings can be seen as holographic effects from higher dimensional geometrical effects in a non-compact 7D Kaluza-Klein surrounding universe [12]: different particles at different spacetime locations enter are in different KK instances and so they don't interact. Higgs boson are located where the particles are, so they can enter the same KK instance. See [12] for more details on this unconstrained KK model. [Note: This paragraph was added in the October 11 version of this paper, the post original write-up.]

With these qualification, it was indeed appropriate to use the inertial mass in the Lagrangian in the previous sections when in the multi-folds.

\section{Non-elementary particles}

For composite particles, like hadrons or atoms, the composite is the particle considered as having paths on the multi-folds and therefore all the arguments above can be repeated and applied to all matter in general.

\section{Conclusions}

This analysis is for a Multi-fold universe as in [1]. [1] details arguments and ways to check its relationship with the real universe. Besides properties that can be experimentally verified (in the future because of the macroscopic weakness of gravity and gravity like effects for entangled systems), [1] shows how the multi-fold mechanisms and behaviors are in many aspects in today's conventional physics, that, at times, anticipate the behaviors modeled in a multi-fold universe. In addition, [1] potentially explains many results obtained in gravity, quantum mechanics, General Relativity, superstring theory, Loop Quantum Gravity and the AdS/CFT correspondence conjecture. All these works attempt to come up with models for the real universe. It is at least a good sign that [1] may provide an interesting model of the real universe. Compilation of outcome and derivative from [1] can be found in [13].

Our analysis has no equivalent or variations for non multi-fold universe: derivation of the equivalence principle is directly tied to the multi-fold mechanisms.

We believe that [1] makes a compelling case for the consistency of its multi-fold proposal. The present paper shows that the weak equivalence principle can be derived in multi-fold universe. The other principles applies to massless, and to massive gravity, when within the range of the latter. Extensions to gravity like effects due to other entanglements is on a case by case basis. The analysis applies to all matter.

The qualification of the multi-fold tenancy model to allow interactions with Higgs bosons within multi-folds is important as normal for multi-fold mechanisms and implied by the reasoning proving the equivalence principle. 
References: (most references come from popular science to make the discussion more approachable) [1]: Stephane H. Maes, (2020) “Quantum Gravity Emergence from Entanglement in a Multi-Fold Universe", viXra:2006.0088v1, (June 9, 2020).

[2]: https://en.wikipedia.org/wiki/Reissner\%E2\%80\%93Nordstr\%C3\%B6m_metric

[3]: https://en.wikipedia.org/wiki/Kerr-Newman metric

[4]: Burinskii, Alexander, (2008), "The Dirac-Kerr-Newman electron", arXiv:0507109v4

[5]: https://en.wikipedia.org/wiki/Equivalence principle

[6]: S. Weinberg, (1964), "Derivation of Gauge Invariance and The Equivalence Principle from Lorentz Invariance and the S-Matrix", Phys. Letters, Vol 9, N. 4

[7]: S. Weinberg, (1965), "Photons and. Gravitons in Perturbation Theory: Derivation of Maxwell's and Einstein's Equations", Phys. Rev., Vol 138, N. 4B

[8]: Richard Feynman, (2002), "Feynman Lectures On Gravitation", Westview Press; 1 edition (June 20, 2002)

[9]: M. Fierz, Wolfgang Ernst Pauli, (1939), "On relativistic wave equations for particles of arbitrary spin in an electromagnetic field", Proc. R. Soc. Lond. A173211-232

[10]: Stephane H Maes, (2020), "Gravity-like Attractions and Fluctuations between Entangled Systems?", viXra:2010.0010v1, https://shmaesphysics.wordpress.com/2020/06/25/gravity-like-attractions-and-fluctuationsbetween-entangled-systems/, June 24, 2020.

[11]: Stephane H Maes, (2020), "Explaining Dark Energy, Small Cosmological Constant and Inflation Without New Physics?", viXra:2006.0261v1, https://shmaesphysics.wordpress.com/2020/06/19/explaining-dark-energy-smallcosmological-constant-and-inflation-without-new-physics/, June 19, 2020.

[12]: Stephane H Maes, (2020), "Tracking Down The Standard Model With Gravity In Multi-Fold Universes", https://shmaesphysics.wordpress.com/2020/08/30/tracking-down-the-standard-model-with-gravityin-multi-fold-universes/, August 20, 2020.

[13]: Stephane H. Maes, (2020), "Web Site Tracking all Publications around the Multi-fold universe" -Navigation page listing all papers. https://shmaesphysics.wordpress.com/shmaes-physics-site-navigation/ 